\title{
Lentivirus-mediated RNA interference targeting WWTR1 in human colorectal cancer cells inhibits cell proliferation in vitro and tumor growth in vivo
}

\author{
JIE PAN, SHAOTANG LI, PAN CHI, ZONGBIN XU, XINGRONG LU and YING HUANG \\ Department of Colorectal Surgery, Affiliated Union Hospital of Fujian Medical University, Fuzhou, Fujian, P.R. China
}

Received December 2, 2011; Accepted February 10, 2012

DOI: $10.3892 /$ or.2012.1751

\begin{abstract}
WW domain-containing transcription regulator 1 (WWTR1) was initially identified as a transcriptional coactivator involved in the differentiation of stem cells as well as the development of multiple organs. Recently, WWTR1 has also been identified as a major component of the novel Hippo signalling pathway important for the development of breast and lung cancer. Here, we show for the first time that WWTR1 has an oncogenic function in colorectal cancer cell lines. Knockdown of WWTR1 by lentivirus-mediated RNA interference in human colorectal cancer cells significantly decreased cell proliferation and the colony formation of RKO cells in vitro and tumor growth in vivo. Furthermore, we found that the decreased proliferation was due to cell cycle arrest and increased apoptosis. In addition, efficient knockdown of WWTR1, demonstrated by quantitative real-time PCR, led to upregulation of ASNS and downregulation of $S M A D 3, L T B R, B A X$ and $B A K 1$ in WWTR1 knockdown cells, suggesting that these genes may be involved in the repression of cell proliferation. Our findings indicate that WWTR1 is an oncogene and has an important role in the proliferation of colorectal cancer cells and in tumor growth in vivo.
\end{abstract}

\section{Introduction}

Colorectal cancer is one of the most prevalent malignancies worldwide. Despite the improvement in its prognosis and therapy in the last few decades, there is presently no efficient cure for disseminated colorectal cancer and nearly half of colorectal cancer tumors have aggressive regrowth of the tumor mass replete with metastatic disease after curative surgery or chemotherapy (1). It is essential to develop new targets and therapeutic approaches. Therapeutic target development requires

Correspondence to: Dr Xingrong Lu, Department of Colorectal Surgery, Affiliated Union Hospital of Fujian Medical University, Fuzhou, Fujian 350001, P.R. China

E-mail: lynxlxr18@163.com

Key words: colorectal cancer, WW domain-containing transcription regulator 1, proliferation, tumorigenicity, cell cycle, apoptosis identification of novel functional molecules, knowledge of their mechanisms of action and strategies for intervention (2).

WW-domain containing transcription regulator 1 (WWTR1), also called TAZ (transcriptional co-activator with PDZ binding motif), activates many transcriptional factors and has important roles in the development of various tissue in mammals (3). WWTR1 has also been shown to regulate stem cell differentiation and self-renewal through binding with the transcription factors PPAR $\gamma, \operatorname{Runx} 2$ and Smad $(4,5)$. Most recently, enhanced expression of WWTR1 has been found in both breast and lung cancer cell lines $(6,7)$. Moreover, overexpression of WWTR1 has been shown to cause enhanced cell proliferation and migration, transformation and the epithelialmesenchymal transition (EMT) in human immortalized mammary epithelial cells, whereas knockdown of WWTR1 in breast cancer cells inhibits tumor formation (7), suggesting that WWTR1 is a novel oncogene and may have important roles in the development of breast cancer. However, whether WWTR1 is also involved in the tumorigenesis of colorectal cancer has not been explored.

In this study, we demonstrate that WWTR1 is important to colorectal cancer. Knockdown of WWTR1 by lentivirusmediated RNA interference in colorectal cancer cells strongly repressed cell proliferation in vitro and tumor growth in vivo, suggesting that WWTR1 is a novel oncogene in colorectal cancer cells.

\section{Materials and methods}

Cell culture. Human colorectal adenocarcinoma cell lines RKO and HCT116 [American Type Culture Collection (ATCC), CRL-2577 and CCL 247) were maintained in RPMI and DMEM, respectively. Both media were supplemented with $10 \%$ fetal calf serum (Omega Scientific, Tarzana, CA), $1 \%$ nonessential amino acids, $100 \mu \mathrm{g} / \mathrm{ml}$ streptomycin, $100 \mathrm{U} / \mathrm{ml}$ penicillin and $3.44 \mathrm{mg} / \mathrm{ml} \mathrm{L-glutamine.} \mathrm{Unless} \mathrm{otherwise}$ indicated, media components were from Gibco-BRL, Life Technologies, Inc. (Grand Island, NY) and chemicals were from Sigma Chemicals (St. Louis, MO). Both cell lines were cultured at $37^{\circ} \mathrm{C}$ in a humidified incubator containing $5 \% \mathrm{CO}_{2}$.

RNA interference construction. Short-hairpin RNAs (shRNAs) specifically targeting human WWTR1 were designed based on 
Table I. Primer sequences used for real-time PCR.

\begin{tabular}{|c|c|c|}
\hline Gene symbol & Primer sequence & Amplicon size (bp) \\
\hline \multirow[t]{2}{*}{ WWTR1 } & Forward: TGCGTGAGAGGATTGAGTTTC & 269 \\
\hline & Reverse: CTTATTGGCGTTGAGGTATGC & \\
\hline \multirow[t]{2}{*}{ ASNS } & Forward: ACGCTGACCCACTACAAG & 138 \\
\hline & Reverse: ACCCAAGTTAGCCTGAGTT & \\
\hline \multirow[t]{2}{*}{ ID1 } & Forward: AGAGACTTTAGGGGGTGGGA & 89 \\
\hline & Reverse: TGAGAAGCACCAAACGTGAC & \\
\hline \multirow[t]{2}{*}{ BAK1 } & Forward: GACGACATCAACCGACGC & 160 \\
\hline & Reverse: AGCCGAAGCCCAGAAGAG & \\
\hline \multirow[t]{2}{*}{ SMAD3 } & Forward: GACTACAGCCATTCCATCC & 201 \\
\hline & Reverse: CAGGTCCAAGTTATTATGTGC & \\
\hline \multirow[t]{2}{*}{ LTBR } & Forward: CTCCTTGCCACCGTCTTCT & 156 \\
\hline & Reverse: CAAGTCAGGGAAGTATGGATGG & \\
\hline \multirow[t]{2}{*}{ BTC } & Forward: GAAATGGAAACTCTGGGT & 126 \\
\hline & Reverse: CAAATGAGCAAGGCACT & \\
\hline \multirow[t]{2}{*}{$\mathrm{BAX}$} & Forward: TGCTTCAGGGTTTCATCCA & 296 \\
\hline & Reverse: GGCCTTGAGCACCAGTTT & \\
\hline \multirow[t]{2}{*}{ GAPDH } & Forward: TGACTTCAACAGCGACACCCA & 121 \\
\hline & Reverse: CACCCTGTTGCTGTAGCCAAA & \\
\hline
\end{tabular}

the GenBank information for WWTR1 (NM_015472). Multiple RNAi candidate target sequences were designed and the one (5'-GCCGATGTGTGAGAAGC-3') having the best interference efficiency was used to knockdown the endogenous WWTR1 in RKO and HCT116 cells. Two DNA oligonucleotides were synthesized as following: WWTR1 -1: 5'-CCGGGAGGTAC TTCCTCAATCACATTTCAAGAGA $\underline{\text { ATGTGATTGAGGA }}$ AGTACCTCTTTTTG-3', WWTR1 -2: 5'-AATTCAAAAAG AGGTACTTCCTCAATCACATTCTCTTGAAATGTGATT GAGGAAGTACCTC-3' (underlined sequences indicate stem sequences). The two oligos were annealed to duplex DNAs in annealing buffer (10 mM Tris, $\mathrm{pH} 8.0,50 \mathrm{mM} \mathrm{NaCl}, 1 \mathrm{mM}$ EDTA). Duplex DNAs were cloned to the lentiviral vector pGCSIL-GFP (GeneChem, Shanghai, China) and transformed to DH5 $\alpha$-competent $E$. coli cells. The positive clones were verified by DNA sequencing. The lentiviral vector pGCSILGFP-Negative (GeneChem) containing an oligonucleotide having no homology with human or mouse genomes was used as a negative control to monitor non-specific responses caused by heterologous siRNAs.

Lentivirus packaging and cell infection. WWTR1 RNAi lentiviral expression vector and control vector were co-transfected with the packaging vectors pHelper1.0 and pHelper2.0 in HEK293T cells according to the instructions for Lipofectamine 2000 (Invitrogen). At $8 \mathrm{~h}$ post-transfection, the medium was replaced to RKO or HCT116 complete medium. On the same day, RKO and HCT116 cells were seeded in $10-\mathrm{cm}$ dishes to $30-50 \%$ confluence. The lentivirus supernatant in HEK293T cells was harvested and the medium in RKO and HCT116 cells was replaced $24 \mathrm{~h}$ later. The medium was replaced to fresh complete medium the next day. The infection efficiency were estimated by checking the GFP-expressing cells under a fluorescence microscope. Cells infected with pGCSIL-GFP negative vector were negative control (NC) cells; cells infected with pGCSIL-GFP-si-WWTR1 vector were knockdown (KD) cells.

Reverse transcription and quantitative real-time polymerase chain reaction. Total RNA was isolated and purified using TRIzol (Invitrogen). Single-stranded cDNA synthesis was performed using the cDNA synthesis kit (Fermentas, USA) according to manufacturer's instructions. The quantification of mRNA levels was carried out using the Thermal Cycler Dice $^{\text {TM }}$ Real-Time PCR System (Takara, Japan). Expression of WWTR1 or the target genes was normalized by an endogenous housekeeping gene, GAPDH. Primers used for PCR are shown in Table I. PCR amplification was programmed for $15 \mathrm{sec}$ at $95^{\circ} \mathrm{C}$, followed by 40 cycles of $95^{\circ} \mathrm{C}$ for $5 \mathrm{sec}$ and $60^{\circ} \mathrm{C}$ for $30 \mathrm{sec}$. Experiments were performed in triplicate in the same reaction. For relative quantification, the expression levels of genes were calculated based on the formula of $2^{-\Delta \Delta C t}$.

Proliferation assay. The proliferation of KD cells and NC cells were examined based on the expression of GFP. Cells were seeded in 96-well plates at a density of 2,000 cells/well. Cells expressing GFP were imaged and analyzed quantitatively using the Cellomics ArrayScan high content screening (HCS) Reader (Thermo Fisher Scientific). Cells were imaged daily for 5 days. The proliferation rate was presented as the fold change of cell numbers at each day compared to Day 1.

Colony formation assay. Cells in medium with $1 \%$ serum were seeded onto 6-well plates at a density of 500-1,000 cells/well and allowed to grow for 14 days to form colonies. Colony cells were fixed with methanol and stained with $0.1 \%$ crystal violet. 


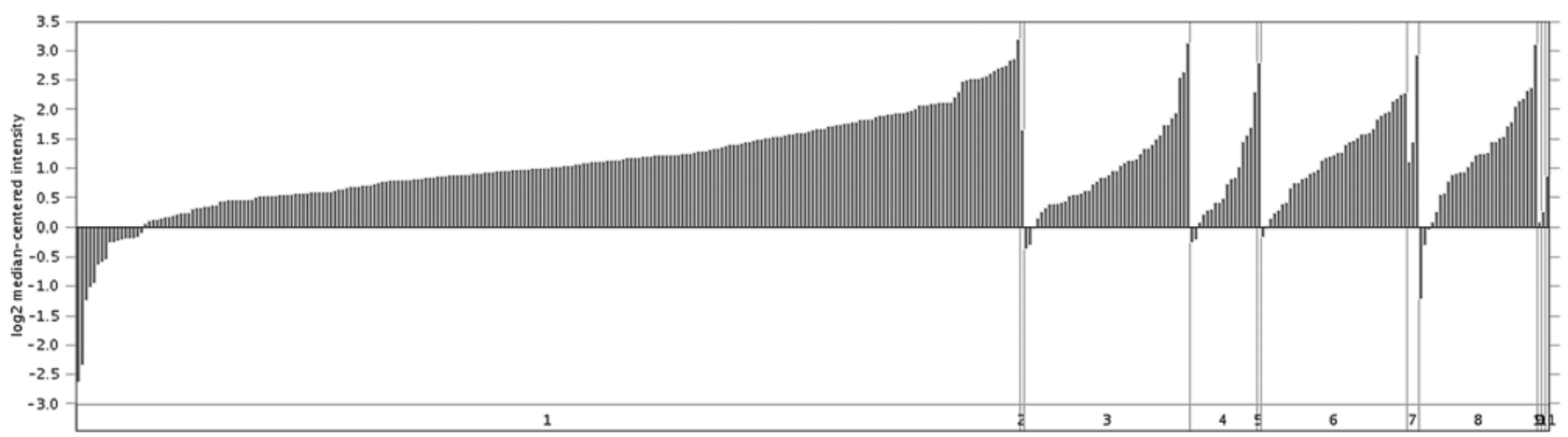

Figure 1. The WWTR1 gene is highly expressed in diverse types of colorectal cancers. 1, Colorectal adenocarcinoma ( $\mathrm{n}=239) ; 2$, colorectal medullary carcinoma $(n=1) ; 3$, colorectal mucinous adenocarcinoma $(n=42) ; 4$, colorectal villous adenoma $(n=17) ; 5$, colorectal signet ring cell adenocarcinoma $(n=1) ; 6$, rectal adenocarcinoma $(n=37) ; 7$, rectal mucinous adenocarcinoma $(n=3) ; 8$, rectosigmoid adenocarcinoma $(n=30) ; 9$, stage 0 colorectal cancer $(n=1) ; 10$, stage 0 colorectal cancer $(n=1) ; 11$, colorectal undifferentiated carcinoma $(n=1)$. The image was downloaded from Oncomine $(8)$.

Visible colonies were counted manually. The rate of colony formation was calculated with the following formula: colony formation rate $=($ no. of colonies/no. of seeded cells) $\mathrm{x} 100 \%$.

Tumorigenicity assay. The effect of knockdown of WWTR1 on tumorigenicity was assessed by subcutaneous injection of $\mathrm{NC}$ cells and KD cells into severe combined immune deficiency (SCID) BALB/c mice. Each aliquot of $5.0 \times 10^{6}$ cells were injected into the back of mice (6-7 weeks old). Mice were maintained under pathogen-free conditions. The formation of subcutaneous tumor was monitored and measured with a digital caliper. The tumor volume was calculated by the following formula: $\mathrm{V}=0.4 \mathrm{xDxd}^{2}(\mathrm{~V}$, volume; $\mathrm{D}$, longitudinal diameter; d, latitudinal diameter). For all the animal experiments, animal handling and experimental procedures were approved by the Animal Experimental Ethics Committee of Fujian Medical University.

Cell cycle analysis. Cultured RKO cells in 6-well plate were harvested by trypsinization and suspended in phosphate-buffered saline (PBS). Cells were centrifuged and fixed in ice-cold $70 \%$ ethanol at $4^{\circ} \mathrm{C}$ for $1 \mathrm{~h}$. After centrifugation and two washes with PBS, cells were resuspended in $1 \mathrm{ml}$ PBS containing $500 \mathrm{U} / \mathrm{ml}$ RNase. Incubation was continued for $30 \mathrm{~min}$ at $37^{\circ} \mathrm{C}$. Cellular DNA was stained with of $20 \mu \mathrm{g} / \mathrm{ml}$ propidium iodide (PI) for 30 min at room temperature in the dark. DNA content was analyzed on a FACSCalibur flow cytometer (BD Biosciences, San Jose, CA, USA) using the CellQuest software. The PI fluorescence signal at FL2A peak vs. the count was used to discriminate $\mathrm{G} 2 / \mathrm{M}$ cells from $\mathrm{G} 0 / \mathrm{G} 1$ doublets. The relative proportions of cells in the G0/G1, S and G2/M phases of the cell cycle were determined from the flow cytometry data.

BrdU incorporation assay. RKO KD cells and NC cells were cultured for 1 and 4 days, respectively. Cells were incubated with 5-bromo-2'-deoxyuridine (BrdU) for $30 \mathrm{~min}$ at $37^{\circ} \mathrm{C}$. After another incubation of $4 \mathrm{~h}$, cells were fixed, washed and incubated with primary and secondary antibodies following the instruction of the BrdU Cell Proliferation assay (Chemicon International Inc., Temecula, CA, USA). The immune complexes were detected by the subsequent substrate reaction and quantitated by measuring the absorbance at $490 \mathrm{~nm}$.
Apoptosis assay. To assess apoptosis, cells were stained with fluorescein isothiocyanate (FITC)-labeled Annexin V and simultaneously with PI to discriminate the intact cells $\left(\right.$ Annexin $\left./ \mathrm{PI}^{-}\right)$from the apoptotic $\left(\right.$Annexin $\left.{ }^{+} / \mathrm{PI}^{-}\right)$and the necrotic cells $\left(\right.$ Annexin $\left.{ }^{+} / \mathrm{PI}^{+}\right)$. A total of $1 \times 10^{6} \mathrm{RKO}$ cells were washed twice with ice-cold PBS and incubated for $30 \mathrm{~min}$ in binding buffer $(1 \mu \mathrm{g} / \mathrm{ml}$ PI and $1 \mu \mathrm{g} / \mathrm{ml}$ FITC-labeled Annexin V). FACS analysis for Annexin V and PI staining was performed by the flow cytometer mentioned previously and the CellQuest software for the detection of apoptosis.

Statistical analysis. All the experiments were repeated three times. The experimental data are presented as mean \pm standard deviation (SD). One-way ANOVA was used for the comparisons among the means. All the analyses were carried out using the SPSS13.0 software (SPSS Inc., Chicago, IL, USA). P-values $<0.05$ were considered to indicate statistically significant differences.

\section{Results}

Expression of WWTR1 in colorectal cancer. In order to assess whether WWTR1 has a function in colorectal cancer, we evaluated its expression pattern in colorectal cancer by mining the cancer microarray database Oncomine (http//www.oncomine. org) (8). Microarray profiling of the gene expression in 373 colorectal cancer tissue samples showed extensive and high expression of WWTR1 in all 11 kinds of colorectal cancer types (Fig. 1), indicating that WWTR1 has the potential to be important in colorectal cancer development.

Knockdown of WWTRI by lentivirus-mediated RNA interference. To explore the possible role of WWTR1 in colorectal cancer, we performed a loss of function assay using RNA interference. Lentivirus vectors that expressed the WWTR1specific siRNA (knockdown, KD) and control siRNA (negative control, NC) were generated and infected into RKO and HCT116 cells. To determine the silencing efficiency, the expression levels of WWTR1 mRNA were detected by realtime PCR. WWTR1 mRNA levels declined significantly in the KD group of both RKO and HCT116 cells, with an average inhibition rate of $80 \%$ compared to NC cells $(\mathrm{P}<0.01)$ (Fig. 2). 

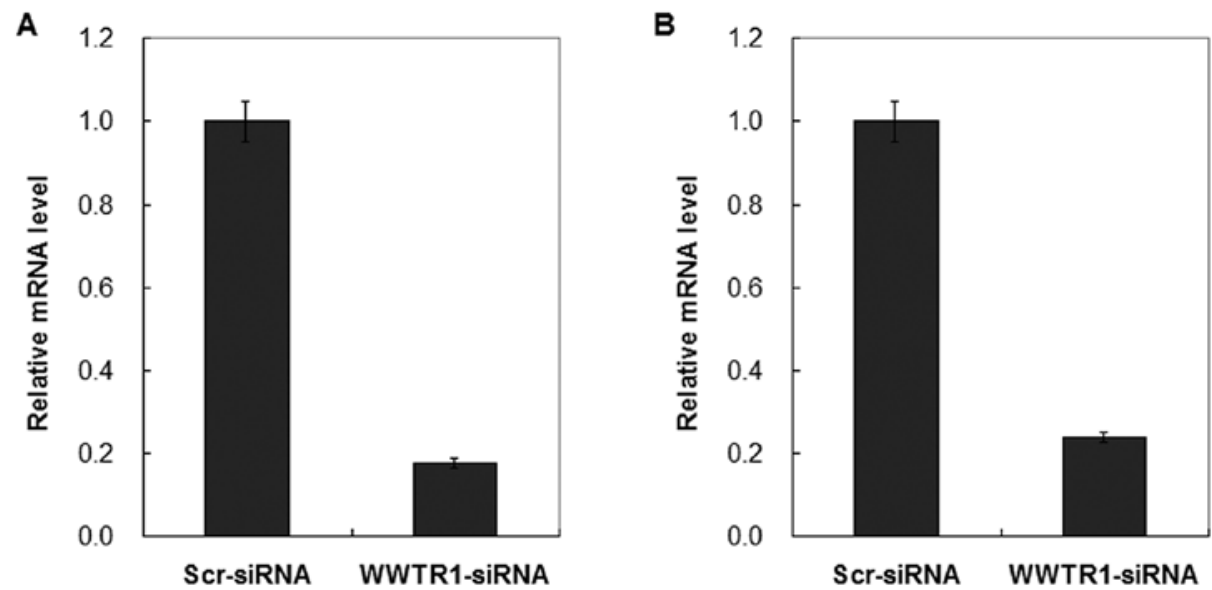

Figure 2. Real-time PCR analysis of the knockdown of WWTR1 by lentivirus-mediated RNA interference in (A) RKO cells and (B) HCT116 cells.
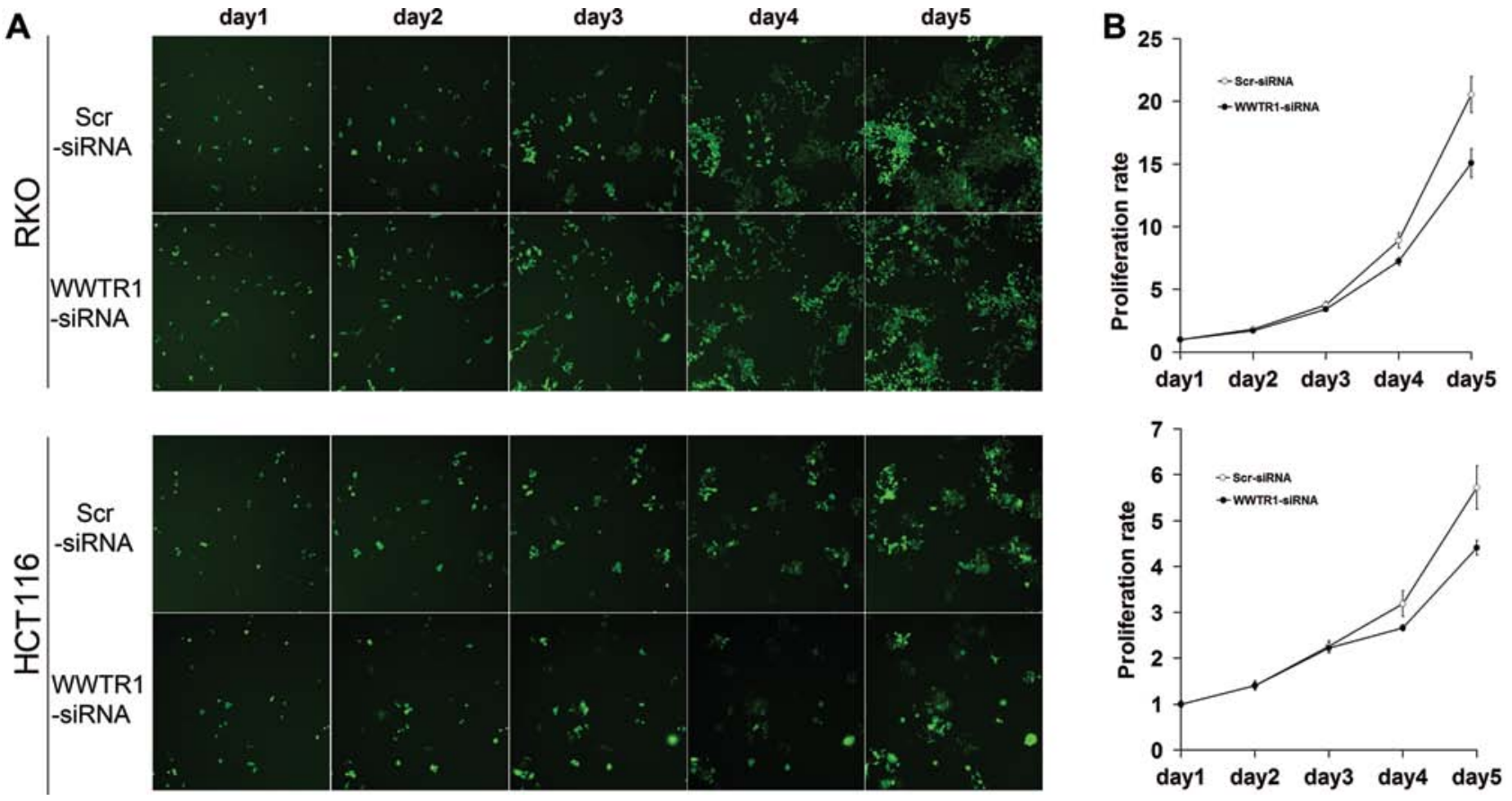

Figure 3. Knockdown of WWTR1 represses proliferation in colorectal cancer cells. (A) Representive Cellomics images of RKO cells and HCT116 cells on Days 1-5. (B) Proliferation curves of RKO cells (upper graph) and HCT116 cells (lower graph). The proliferation rates are presented as the fold changes of the cell number at each time point compared to Day 1. Each value represents the mean of three replicates.

Thus, WWTR1-specific siRNA could efficiently downregulate WWTR1 expression.

WWTR1 knockdown represses cell proliferation. Deregulated cell growth appears in many types of cancer and is regarded as an important characteristic of cancer. The effects of WWTR1 knockdown on the proliferation of RKO and HCT116 cells were examined daily using the Cellomics ArrayScan high content screening (HCS) platform. Strikingly, both RKO and HCT116 KD cells showed decreased proliferative capacity on Day 4 compared to the NC cells, and this effect increased on Day 5. The differences between the groups were statistically significant $(\mathrm{P}<0.01)$ (Fig. 3), suggesting that knockdown of WWTR1 led to decreased proliferation in colorectal cancer cells.
Knockdown of WWTR1 represses colony formation in vitro and tumor growth in vivo. Colony formation is a hallmark of malignancy of cancer cells in vitro (9). The effect of knockdown of WWTR1 on the colony formation in RKO cells was examined. As shown in Fig. 4A and B, RKO KD cells showed significantly fewer colonies than NC cells $(\mathrm{P}<0.05)$, indicating the decreased ability of single KD cells to form colonies compared to NC cells.

To determine the role of WWTR1 in the tumorigenicity in vivo, a xenograft tumor model was established in BALB/c nude mice. RKO NC cells developed visible subcutaneous tumors about 2 weeks post-inoculation and grew rapidly in the next 3 weeks. In contrast, KD cells developed tumors slowly and the tumors were visible only at 4 weeks post-inoculation, 

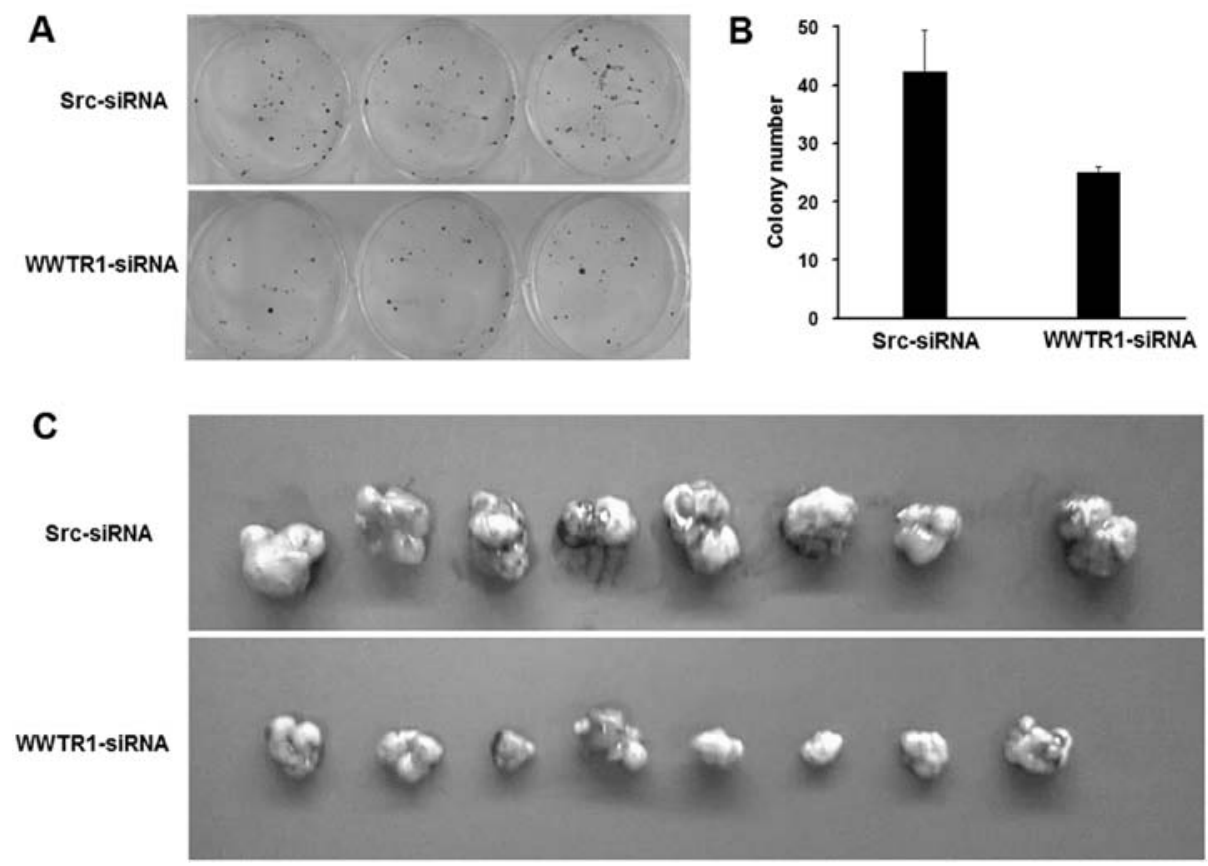

Figure 4. Knockdown of WWTR1 affects maligancy of RKO cells. (A) A decreased number of colonies was formed in RKO KD cells compared to NC cells. (B) In the xenograft model, RKO KD cell-derived tumors were much smaller than RKO NC cell-derived tumors.
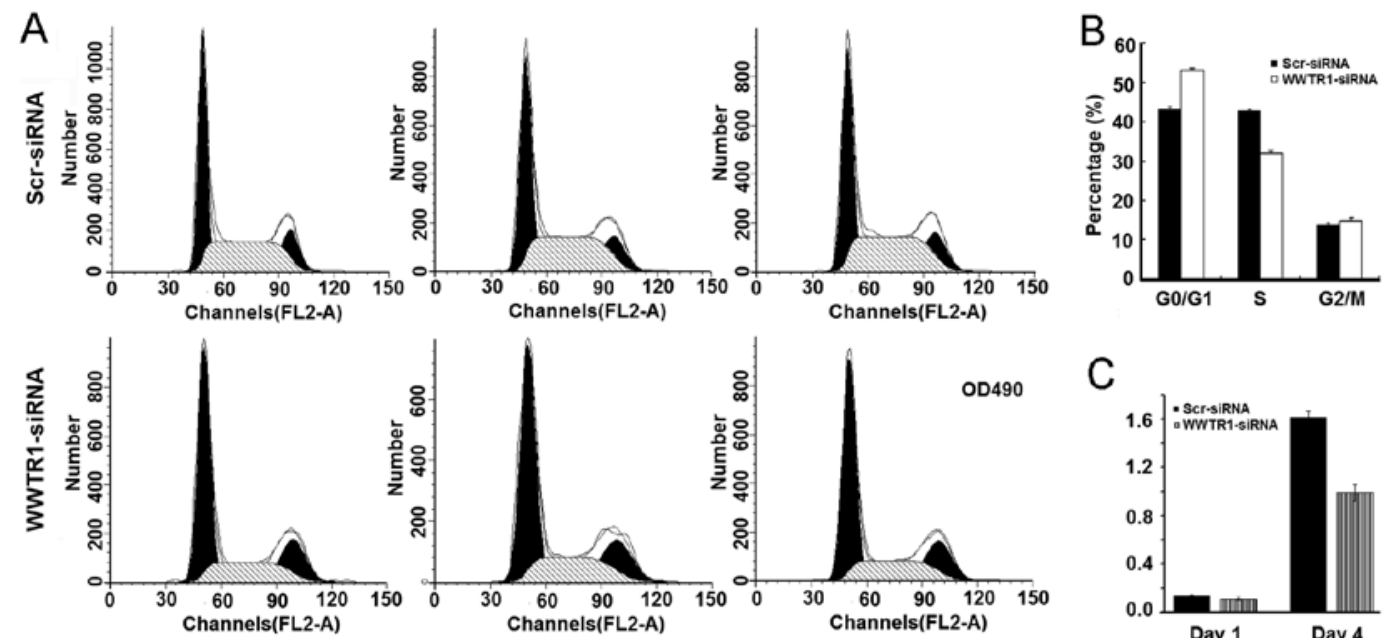

C
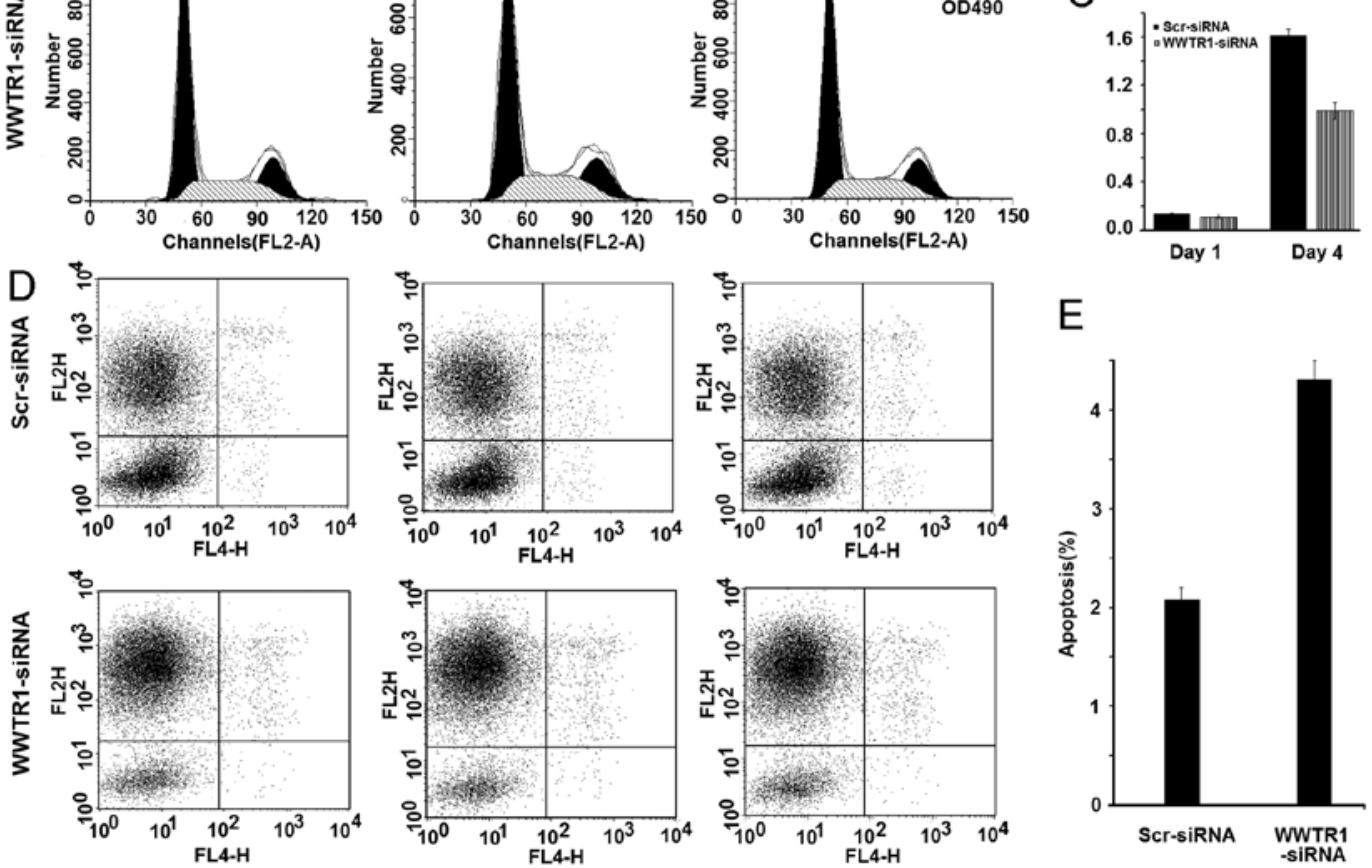

Figure 5. Influence of the knockdown of WWTR1 on cell cycle progression, cell division and apoptosis. (A) Analysis of cell cycle progression of RKO KD and NC cells was performed using FACS. (B) Percentages of cells at different cell cycle phases in RKO cells. The mean percentage of S-phase cells was lower $(\mathrm{P}<0.05)$ and that of $\mathrm{G} 0 / \mathrm{G} 1$ phase cells was higher $(\mathrm{P}<0.05)$ in the KD cells than in the NC cells. (C) BrdU incorporation assay. RKO KD cells have higher BrdU incorporated DNA content than NC cells ( $\mathrm{P}<0.05)$. (D) Analysis of cell apoptosis of RKO KD and NC cells was performed using a flow cytometer. (E) Percentages of apoptotic cells in RKO cells. The mean percentage of apoptotic cells was higher $(\mathrm{P}<0.05)$ in the KD cells than in the NC cells. 

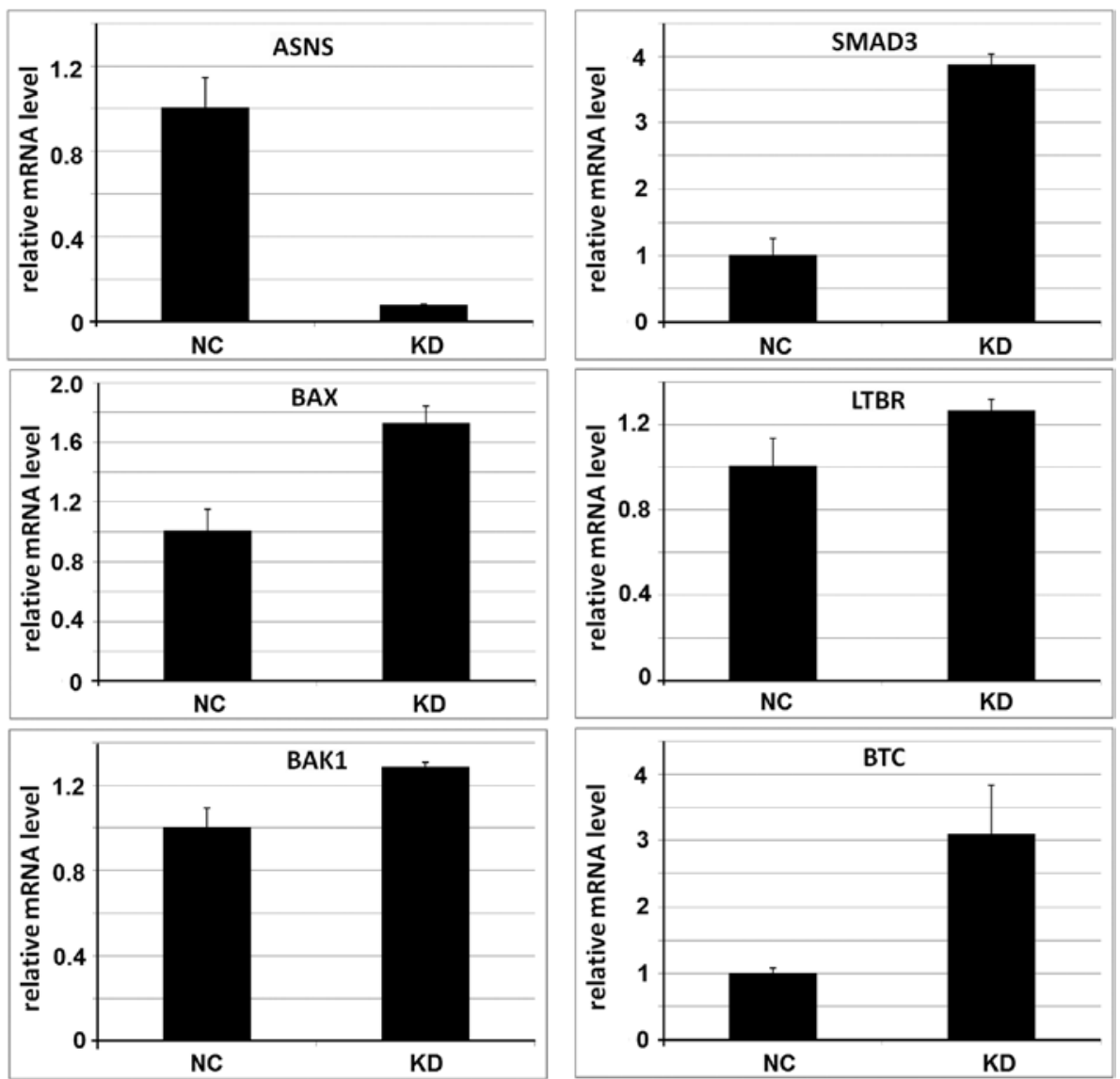

Figure 6. Examination the expression of selected genes by real-time PCR. Expression of the selected genes was normalized to GAPDH. The relative gene expression is represented as the fold change in $\mathrm{KD}$ cells over that in NC cells.

and the average volume of tumors in the KD group was significantly smaller than that in the NC group after 6 weeks of inoculation $(\mathrm{P}<0.01)$ (Fig. 4C). Collectively, these data demonstrated that lentivirus mediated RNAi targeting of WWTR1 significantly inhibited tumor growth of RKO cells in vivo.

Knockdown of WWTRl induces cell cycle arrest. To ascertain whether the decreased cell proliferation induced by knockdown of WWTR 1 is due to cell cycle arrest, we determined the cell cycle distribution in RKO KD and NC cells. Flow cytometric analysis revealed that the G0/G1-phase DNA content was higher in KD cells $(53.61 \%)$ than in NC cells $(43.3 \%)(\mathrm{P}<0.05)$, while the S-phase DNA content was lower in KD (31.7\%) than in $\mathrm{NC}$ cells $(42.9 \%)(\mathrm{P}<0.05)$ (Fig. $5 \mathrm{~A}$ and $\mathrm{B})$, indicating that more KD than NC cells showed cell cycle arrest.

We further measured the cell dividing rate by the BrdU incorporation assay. Dividing cells can be labelled by BrdU during DNA synthesis. As shown in Fig. 5C, the rate of DNA synthesis in RKO KD cells was not affected on Day 1 of infection, but was significantly decreased on Day 4 compared to the $\mathrm{NC}$ group $(\mathrm{P}<0.05)$ (Fig. 5C). These data indicate that downregulation of WWTR1 induced G0/G1 phase cell cycle arrest and decreased cell division, which contributed to the inhibition of cell growth and proliferation of RKO KD cells.

Knockdown of WWTRl enhances cell apoptosis. It is generally accepted that cancer does not necessarily arise as a result of cell proliferation increase. Rather, there is an essential balance between the rate of cell-cycle progression and cell apoptosis (10). To examine whether the reduced cell proliferation in WWTR1 knockdown cells was due to increased cell apoptosis, the rate of apoptosis was evaluated using Annexin V/PI double staining-coupled flow cytometry analysis. As shown in Fig. 5D and E, the apoptosis rate in RKO KD cells (4.34\%) was significantly higher than in RKO NC cells $(2.05 \%)(\mathrm{P}<0.05)$, suggesting that downregulation of WWTR1 significantly increased the population of apoptotic cells. These data demonstrate that suppression of cell proliferation in RKO KD cells may be partly caused by increased apoptotic cell death.

Examination of the target genes of WWTRl in knockdown cells. To understand the underlying mechanism of the anti-proliferative effects observed in cells with WWTR1 knockdown, we selected several potential downstream genes from the published DNA microarray data of WWTR1-overexpressing cells $(11,12)$. The selected genes, ASNS, SMAD3, LTBR, BTC, $B A X, B A K 1$, have been reported to play important roles in the cell cycle or in apoptosis processes (13-20). Quantitative realtime PCR showed that ASNS and SMAD3 were significantly repressed and upregulated in WWTR1 KD cells $(\mathrm{P}<0.01)$ (Fig. 6). Three genes ( $L T B R, B A X, B A K 1)$ were somewhat upregulated in KD cells $(\mathrm{P}<0.05)$, while no change was found for the $B T C$ gene. The downregulation of a proliferationpromoting gene and the upregulation of apoptosis genes in KD cells indicate the possible mechanism for si-WWTR1- induced anti-proliferative effects. 


\section{Discussion}

WWTR1 has been reported to promote cell proliferation and metastasis in breast and lung cancer $(6,7)$. In the current study, our data indicate an oncogenic function for WWTR1 in colorectal cancer. Knockdown of WWTR1 in colorectal cancer cells repressed cell proliferation in vitro and tumorigenicity in vivo. These findings were supported by arrested cell cycle and increased apoptosis in colorectal cancer cells with knockdown of WWTR1. In accordance to these findings, WWTR1 has also been shown to be inhibited by the Hippo tumor suppressor pathway, which contains the well-established human tumor suppressor NF2 and other genes mutated in human cancer, such as Sav and Mob (21).

To explore the underlying mechanism of si-WWTR 1 induced anti-proliferation, we examined the expression of several potential downstream genes in RKO KD cells. As expected for a proliferation activator, we observed downregulation of $A S N S$, which has been described to be one of the genes related to the entrance to the $S$ phase of the cell cycle $(13,14)$. Other upregulated genes included SMAD3, $B A X, B A K$ and $L T B R$. Smad3 has been found to be highly expressed in quiescent cells with a significant drop in proliferating cells (15). Bax mediates Yes kinase-associated protein (YAP)-induced apoptosis by interaction with p73 (19). Bak collaborates with Bax to permeabilize the mitochondrial outer membrane, leading to the release of apoptogenic factors (17). Lymphotoxin- $\beta$ receptor (LT $\beta \mathrm{R}$ ) is critical to induce cell death via caspase-dependent and caspase-independent pathways (20). Among these examined genes, SMAD3 is a well-characterized target of WWTR1. WWTR1 binds heteromeric Smad2/3-4 complexes and is recruited to TGF $\beta$ response elements (5). Bax has also been demonstrated be a target of YAP (19), which is highly homologous and has overlapping functions with WWTR1 (22). WWTR1 itself has no DNA binding domain; therefore, it must bind to DNA binding transcription factors to stimulate downstream target gene expression. It is interesting to identify the direct DNA binding transcription factors that mediate the altered expression of these examined genes in WWTR1 knockdown cancer cells.

In summary, our study identified WWTR1 as an oncogene in colorectal cancer. Knockdown of WWTR1 significantly repressed cell proliferation and tumor growth of colorectal cancer cells. These findings advance our understanding of the role of WWTR1 in colorectal cancer development and provide a potential therapeutic target for human cancer.

\section{Acknowledgements}

We thank Huiming Lin, Shenghui Huang and Yanwu Sun for their valuable suggestions. This study was supported by the National Natural Science Foundation (30872479).

\section{References}

1. Khair G, Monson JR and Greenman J: Epithelial molecular markers in the peripheral blood of patients with colorectal cancer. Dis Colorectal Rectum 50: 1188-1203, 2007.
2. Arumugam T, Ramachandran V and Logsdon CD: Effect of cromolyn on S100P interactions with RAGE and pancreatic cancer growth and invasion in mouse models. J Natl Cancer Inst 98: 1806-1818, 2006

3. Wang K, Degerny C, Xu M and Yang XJ: YAP, TAZ, and yorkie: a conserved family of signal-responsive transcriptional coregulators in animal development and human disease. Biochem Cell Biol 87: 77-91, 2009.

4. Hong JH, Hwang ES, McManus MT, et al: TAZ, a transcriptional modulator of mesenchymal stem cell differentiation. Science 309: 1074-1078, 2005

5. Varelas X, Sakuma R, Samavarchi-Tehrani P, et al: TAZ controls Smad nucleocytoplasmic shuttling and regulates human embryonic stem-cell self-renewal. Nat Cell Biol 10: 837-848, 2008.

6. Chan SW, Lim CJ, Guo K, et al: A role for TAZ in migration, invasion, and tumorigenesis of breast cancer cells. Cancer Res 68: 2592-2598, 2008.

7. Zhou Z, Hao Y, Liu N, Raptis L, et al: TAZ is a novel oncogene in non-small cell lung cancer. Oncogene 30: 2181-2186, 2011.

8. Rhodes DR, Yu J, Shanker K, et al: ONCOMINE: a cancer microarray database and integrated data-mining platform. Neoplasia 6: 1-6, 2004.

9. Park CH, Bergsagel DE and McCulloch EA: Mouse myeloma tumor stem cells: a primary cell culture assay. J Natl Cancer Inst 46: 411-422, 1971.

10. Reed JC: Dysregulation of apoptosis in cancer. J Clin Oncol 17: 2941-2953, 1999.

11. Zhang H, Liu CY, Zha ZY, et al: TEAD transcription factors mediate the function of TAZ in cell growth and epithelialmesenchymal transition. J Biol Chen 284: 13355-13362, 2009.

12. Lai D, Ho KC, Hao Y and Yang X: Taxol resistance in breast cancer cells is mediated by the Hippo pathway component TAZ and its downstream transcriptional targets Cyr61 and CTGF. Cancer Res 71: 2728-2738, 2011.

13. Colletta G and Cirafici AM: TSH is able to induce cell cyclerelated gene expression in rat thyroid cell. Biochem Biophys Res Commun 183: 265-272, 1992.

14. Krejci O, Starkova J, Otova B, et al: Upregulation of asparagine synthetase fails to avert cell cycle arrest induced by L-asparaginase in TEL/AML1-positive leukaemic cells. Leukemia 18: 434-441, 2004.

15. Daly AC, Vizán P and Hill CS: Smad3 protein levels are modulated by Ras activity and during the cell cycle to dictate transforming growth factor- $\beta$ responses. J Biol Chem 285: 6489-6497, 2010.

16. Shin HS, Lee HJ, Nishida M, et al: Betacellulin and amphiregulin induce upregulation of cyclin D1 and DNA synthesis activity through differential signaling pathways in vascular smooth muscle cells. Circ Res 93: 302-310, 2003.

17. Brooks C and Dong Z: Regulation of mitochondrial morphological dynamics during apoptosis by Bcl-2 family proteins: a key in Bak? Cell Cycle 6: 3043-3047, 2007.

18. Westphal D, Dewson G, Czabotar PE and Kluck RM: Molecular biology of Bax and Bak activation and action. Biochim Biophys Acta 1813: 521-531, 2011.

19. Zhang H, Wu S and Xing D: YAP accelerates A $\beta(25-35)$-induced apoptosis through upregulation of Bax expression by interaction with p73. Apoptosis 16: 808-821, 2011.

20 . You RI, Chen MC, Wang HW, et al: Inhibition of lymphotoxin- $\beta$ receptor-mediated cell death by survivin- $\Delta \mathrm{Ex} 3$. Cancer Res 66 : 3051-3061, 2006.

21. Zhao B, Wei X, Li W, et al: Inactivation of YAP oncoprotein by the Hippo pathway is involved in cell contact inhibition and tissue growth control. Genes Dev 21: 2747-2461, 2007.

22. Lei QY, Zhang H, Zhao B, et al: TAZ promotes cell proliferation and epithelial-mesenchymal transition and is inhibited by the Hippo pathway. Mol Cell Biol 28: 2426-36, 2008. 